\title{
Provenance and Reproducibility in the Automation of a Standard Computational Neuroscience Pipeline
}

\author{
David Bruce Stockton \\ davidbrucestocktonbme@gmail.com \\ Emory University \\ Atlanta, Georgia
}

\author{
Astrid A. Prinz \\ Emory University \\ Atlanta, Georgia
}

\author{
Fidel Santamaria \\ University of Texas at San Antonio \\ San Antonio, Texas
}

\begin{abstract}
Rapid increase in data volume, compounded by the reproducibility crisis, has led to the need to automate both experimental and computational aspects of neuroscience investigations. Automating neuroscience investigations enables an unprecedented ability to record and inspect how results were achieved. Here we review some of our recent work to integrate provenance and reproducibility measures into a tool called NeuroManager that automates a standard computational neuroscience pipeline, unifying the experimentdata-modeling-analysis cycle and allowing the scientist to focus on model evolution. Through a flexible daily workflow that leverages servers, clusters, and clouds simultaneously, NeuroManager automates manual tasks including database access, job submission, simulation scheduling, and preservation of provenance.
\end{abstract}

\section{CCS CONCEPTS}

- Computing methodologies $\rightarrow$ Simulation tools; $\bullet$ Applied computing $\rightarrow$ Bioinformatics; • Software and its engineering $\rightarrow$ Software configuration management and version control systems; Documentation; Software evolution.

\section{KEYWORDS}

neuroinformatics, provenance, modeling, workflow

\section{ACM Reference Format:}

David Bruce Stockton, Astrid A. Prinz, and Fidel Santamaria. 2019. Provenance and Reproducibility in the Automation of a Standard Computational Neuroscience Pipeline. In 2nd International Workshop on Practical Reproducible Evaluation of Computer Systems (P-RECS '19), Fune 24, 2019, Phoenix, AZ, USA. ACM, New York, NY, USA, 6 pages. https://doi.org/10.1145/3322790. 3330592

\section{INTRODUCTION}

An increasingly common neuroscience research scenario attempts to match results between "wetlab" (in vivo, ex vivo, or in vitro) experimentation with biological specimens, and "drylab" (in silico) experimentation involving computer models and simulations. Computer models are also used to study the statics and dynamics of neural systems in experiments that are impossible or impractical to

Permission to make digital or hard copies of all or part of this work for personal or classroom use is granted without fee provided that copies are not made or distributed for profit or commercial advantage and that copies bear this notice and the full citation on the first page. Copyrights for components of this work owned by others than ACM must be honored. Abstracting with credit is permitted. To copy otherwise, or republish, to post on servers or to redistribute to lists, requires prior specific permission and/or a fee. Request permissions from permissions@acm.org.

P-RECS'19, fune 24, 2019, Phoenix, AZ, USA

(C) 2019 Association for Computing Machinery.

ACM ISBN 978-1-4503-6756-1/19/06 . .\$15.00

https://doi.org/10.1145/3322790.3330592 run in wetlab, such as iterating through a set of neuronal morphologies, or varying the concentration of biophysical entities, such as ion channels or pumps, that may have no specific pharmacological manipulator. Just as wetlab experimental protocol must be recorded and reported in order to establish confidence in and verify reported findings, drylab experimentation must pass the same bar of accurate and comprehensive recording.

Unfortunately, at least half of scientific studies are not reproducible, including computational studies, and there is growing consensus that there is a reproducibility crisis [4]. Researchers in computational neuroscience are taking notice and articulating the need for addressing various facets of reproducibility as part of standard practice [22]. The BRAIN Initiative has as a core principle to "Create and support an infrastructure for preserving and sharing data", with a key deliverable "tools ... that improve reproducibility of published results" [5].

In this paper we isolate and focus on some of the overt, specific measures we took to improve provenance and reproducibility in the design and development of a computational neuroscience tool called NeuroManager that automates much of the experimentdata-modeling-analysis cycle. NeuroManager is discussed fully in multiple publications and summarized below.

\section{THE IMPORTANCE OF PROVENANCE AND REPRODUCIBILITY IN COMPUTATIONAL NEUROSCIENCE}

Although the idea of recording the protocol and specific components of a wetlab experiment is well-known to every student, at the professional scientific level there are many obstacles to performing accurate provenance that leads to reproducible results. Lack of knowledge of wild animal sources, nonuniformity in biochemical supplies, and even the question of identifying research resources properly is an issue [38]. Tracking these details, however, is fundamental to tracing and understanding biological variability across subjects and species.

These factors also affect the reproducibility of a wetlab experiment. Basic problems in reproducibility come from 1) intrinsic biological variability, since no two animals, or neurons, are exactly the same; 2) experimenter functional variability, since even if two people follow the exact same experimental protocol, subtle differences in skills and execution can lead to different outcomes; 3 ) factors that are unknown or considered irrelevant and therefore not controlled, such as time of day, room temperature, laboratory light level, or ambient noise; and 4) experimenter characteristics such as gender or $\operatorname{mood}[9,20]$. 
In contrast, computational neuroscience is subject to few, if any, of these obstacles. Instead, there are other issues to consider, such as numerical accuracy, choice of equation or algorithm, subtle or not so subtle mathematical or coding errors, model verification, software versions, open vs. closed source, reliability and longevity of support, and compatibility between a multiplicity of data formats. Given the computerized nature of the field, one would expect that recording such details would be a well-established part of computational neuroscience. There are efforts at improvement, such as the ReproNim project, which addresses the reproducibility of imaging [19], or the PANDORA toolbox, which seeks to standardize analysis between wetlab and drylab [17]. In general, though, this is a neglected area, partly because of the informal interactive approach which tends to be standard in the field at present and which leads to undocumented changes in algorithms, concentrations, or equations. Encouragingly, computer science is addressing these issues as a subfield common to computational science [12].

\section{THE NEUROMANAGER TOOL}

The NeuroManager tool [34] is an object-oriented software package written in MATLAB (Natick, Mass) that runs computational neuroscience simulations ${ }^{1}$ on remote computational resources and returns the results to the user automatically. A detailed discussion of NeuroManager workflow in Stockton and Santamaria [34] highlights the savings to the user over manual simulation, including file transfer, compilation of model files, and production of output products and analyses. NeuroManager wraps established, domainspecific tools such as the NEURON simulator [18] to create a pool of Virtual Simulators (Figure 1) that are distributed between the Host and a set of Target Machines which can be Linux servers; clusters managed by Sun Grid Engine (SGE) [37] or Simple Linux Utility for Resource Management (SLURM) [39]; cloud servers available through Chameleon [8], Rackspace [25], or Amazon Web Services (AWS) [3]; or any mix thereof [35]. In a NeuroManager Investigation [33], the researcher configures the Simulator with a biological model and creates a Simulation Set (manually or automatically), and then the Simulation Management module schedules those simulations on the Simulator Pool based on a modified min-min strategy. The results of simulations are transferred to the host and entered into a MySQL Investigation Database [23], which is machine or human queryable. The researcher can automatically access databased wetlab results to enable parameter searches and compare wetlab and drylab findings. In particular, we automated use of the Allen Brain Institute Cell Types Database [1]; the wetlab results are also placed into the investigation database, allowing the user to query data, analysis, and comparisons using MySQL queries [36]. The investigation database is stored as a dump file, forming one component of investigation provenance. ${ }^{2}$ The software also keeps an extensive $\log$ that includes software versions, all job submission files, and many other reproducibility-oriented investigation artifacts.

\footnotetext{
${ }^{1}$ The words Simulation, Simulator, Machine, Investigation are specialized concepts and software objects in NeuroManager and if capitalized are used in that way.

${ }^{2}$ Note: at present the investigation database does not include provenance-specific data such as software versions, simulator versions, etc.. That information, however, is available in the files stored in a unique, date/time-stamped directory associated with the investigation. Such features would be highly desirable in future editions.
}

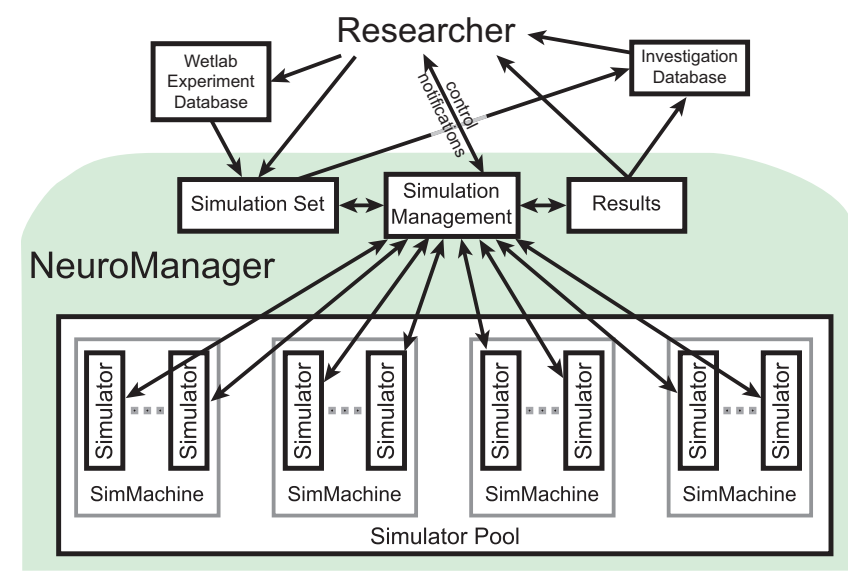

Figure 1: NeuroManager Manifest Architecture. NeuroManager (shaded area) creates a pool of Virtual Simulators that are distributed between the Host computer and Target Machines which can be servers, clusters, or clouds, or any mix thereof. Virtual Simulators are distributed Virtual Machines that wrap a SimCore, which is a command-line neuroscience simulator such as NEURON, MCell, or custom code. In a NeuroManager Investigation, the researcher creates a Simulation Set (manually or automatically) and the Simulation Management module schedules those simulations on simulators based on a modified min-min strategy. The results of simulations are transferred to the host and entered into the Investigation Database, which is machine or human queryable.

Although our development was focused on NEURON, NeuroManager integrated well with MCell [28], and should also integrate with GENESIS [6], BRIAN [15], and other neuroscience simulators. The key requirements for a domain-specific tool to be incorporated into NeuroManager are that it has a command-line interface and runs on LINUX. So we believe that NeuroManager could be useful in other simulation-based domains, particularly computational biology, where provenance and reproducibility are important. NeuroManager is hosted on GitHub under an open source license. ${ }^{3}$ The repository provides an extensive user manual, with addenda, that includes a progressive "Hello, world" tutorial showing increased stages of connectivity and manipulation of models [29, 31-33].

\section{RELATED WORK}

Space restrictions do not permit a full discussion here of work related to NeuroManager; however in associated papers there are solid discussions of related work in computational science focusing on workflow [34], cloud utilization [35], and database incorporation [36].

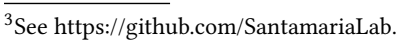




\section{INCORPORATING FLEXIBILITY INTO A FIXED EXPERIMENTAL PIPELINE}

In developing NeuroManager, we adopted a fixed experimental pipeline of nine abstract stages or blocks that matches the flow of many computational neuroscience projects [34]. Having a fixed pipeline improves transparency and reproducibility, and reduces the provenance burden, yet can limit the user's ability to adapt models or leverage varied resources. In contrast, many scientific workflows need more flexibility [10]. We mapped the nine stages into twenty-two actual NeuroManager processes [30, 32], each implemented using object methods that can be supplemented or overridden through the machine class hierarchy to achieve a high degree of flexibility. For example, an SGE cluster permitted model file compilation on cluster nodes, whereas another SLURM cluster did not. Customizing the appropriate object methods allows the daily user to treat the clusters as identical. Similarly, job submission on clusters involves preconstruction of a cluster-dependent job file, while job submission on servers and clouds does not; the "Pre Run Job Processing" method permits the flexibility to treat these cases differently.

\section{CREATING AN INTER-RESOURCE THAT INCLUDES CLOUDS}

Combining multiple independent clouds into one computational resource is known as a multi-cloud, a type of inter-cloud [16]. Since we also added similarly independent clusters and servers, we extend the term to inter-resource. In this sense, then, NeuroManager acts as an inter-resource broker. ${ }^{4}$

The need to place Virtual Simulators on computational resources of varied type, each with varying support for gridware, eliminated many options for leveraging gridware to set up Virtual Simulators and run simulations. In addition, each simulation needed to be scheduled independently for overall performance of an entire simulation set. Accordingly, for cloud interactions we chose to use ground-level routines such as the use of cURL [27] and each cloud's RESTful APIs, SDKs, ${ }^{5}$ and endpoints $[2,26]$ rather than make use of containers such as Docker, ${ }^{6}$ cloud provisioning facilitators such as Ansible, ${ }^{7}$ specific cloud utilities such as Heat, ${ }^{8}$ or features for cluster-based parallelism such as array jobs. ${ }^{9}$

The ground-level approach allowed us to treat the three different computational types as similarly as possible, as well as to write interface classes that masked API operations from NeuroManager core classes. The machine object hierarchy approach was ideal for managing differences between clouds [31]. Handling the multiple encryption levels required for AWS requests, however, exceeded our available development time constraints, and we compromised by limiting AWS use to servers constructed manually. Managing the RESTful interactions for the other two clouds was less involved

\footnotetext{
${ }^{4}$ Singularity (https://singularity.lbl.gov), which began after the original NeuroManage paper was published, might be useful in future versions of NeuroManager. Thank you to our reviewers for this suggestion!)

${ }^{5}$ See https://www.openstack.org/software/project-navigator/sdks.

${ }^{6}$ See https://www.docker.com/.

${ }^{7}$ See https://www.ansible.com/.

${ }^{8}$ See https://wiki.openstack.org/wiki/Heat.

${ }^{9}$ See http://wiki.gridengine.info/wiki/index.php/Simple-Job-Array-Howto.
}

and we were able to incorporate both persistent and ephemeral servers into NeuroManager operation.

\section{ENHANCING REPRODUCIBILITY THROUGH CLOUD SERVER IMAGING}

The use of standalone servers and clusters as computational resources has an inherent problem - the user is dependent on current support and software versions provided by the resource. The user has little sway over versions and almost none over legacy software that may be required for reproduction of a published computational experiment. Cloud servers, however, are not as subject to unrelated trends, and the user configures servers according to need, including custom combinations of software versions. A computational tool like NeuroManager that can exploit this potential [35] is a strong aid in better reproducibility.

In NeuroManager, a user manually creates and tests a cloud server configured with a specific combination of required software, then creates an image file used to automatically create cloud servers with that configuration. To create or employ a single or bank of cloud servers, NeuroManager uses a cloud server information file in JSON format ${ }^{10}$ that specifies the name of the server; a cloud information file (also in JSON format) which contains endpoints, network information, and available images; the name of the image file; and flavor (a cloud term referring to the core/memory configuration of the virtual machine to be used). For example, one image that we used on Chameleon Cloud during development of NeuroManager called "CC-CentOS7-MCR2013a-NEURON74-Python27" was a combination of the CentOS07 operating system ${ }^{11}$, the MCR associated with MATLAB 2013a [21], NEURON 7.4, and Python 2.7. An extensive discussion of cloud terms and provenance using virtual machines can be found in Stockton and Santamaria [35].

\section{ISOLATING AND COUPLING SIMULATION-SPECIFIC INFORMATION FOR CORRECTNESS AND PROVENANCE}

When handling multiple simulations, it is important to ensure that the information that distinguishes them is kept isolated and that related provenance is well coupled [7]. We used specific mechanisms at the Investigation, Machine, and Simulation levels to pass simulation-specific information to generalized processors in an isolated way. For machines and simulations, our basic approach was to have a user-customizable function called "userSimulation" that was incorporated into the remotely-hosted Virtual Simulator code and is called with seven input string parameters that automatically change with machine and simulation. The seven parameters are: machine ID (the machine that particular Virtual Simulator is running on), simulation ID, run directory (the machinespecific directory the simulation will be running in), input directory (machine-specific; where all input files will be found), model directory (machine-specific; where all model files will be found), output directory (machine-specific; where all output files will be placed), and varargin, a list of ten string parameters associated with the simulation and defined in the Simulation Set. In this

\footnotetext{
${ }^{10}$ JSON' stands for Javascript Object Notation. Please see http://www.json.org/.

${ }^{11}$ See https://www.centos.org/.
} 


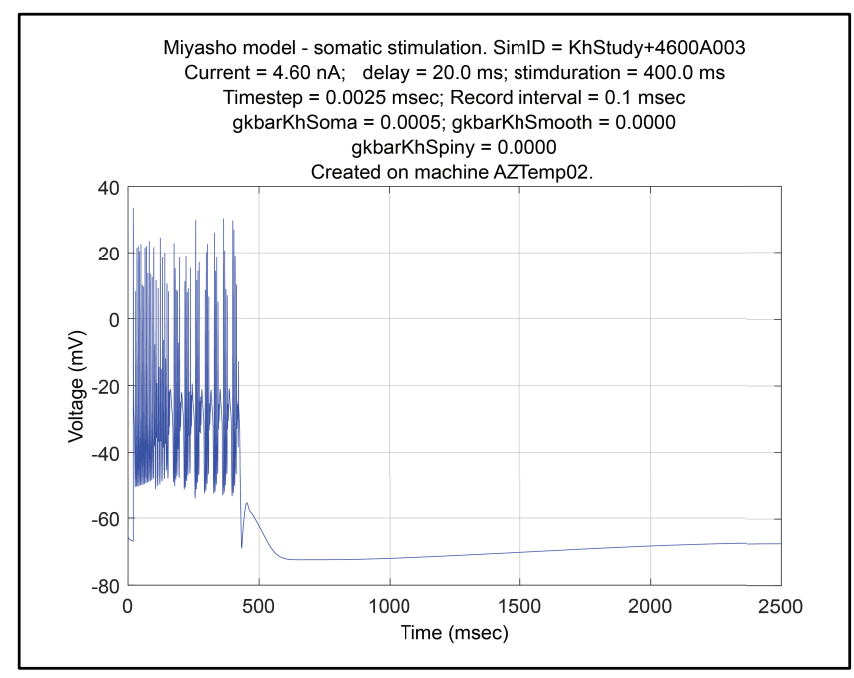

Figure 2: Isolation and coupling of simulation-specific data. This plot, produced automatically on computational resource AWS emphemeral cloud server AZTemp02, shows the automatic passing of simulation input parameters to the simulation, then to the figure title, ensuring accurate provenance of the figure. Once the output results are transferred to the host computer to a newly-created time/datestamped directory, the remote simulation's files and directory structure are wiped, ensuring that there is no transfer of any file to the following simulation. The Miyasho model can be found on ModelDB, at https://senselab.med.yale.edu/ ModelDB/ShowModel.cshtml? model=17664.

function, then, the user makes use of these string parameters without knowing what their specific values are or what machine the simulation will be running on. By using these parameters in the production of output text and graphics, the user avoids all cut-andpaste errors and ensures correctly isolated and coupled provenance for each output product (Figure 2). Just as input parameters are quarantined from one another using this approach, NeuroManager also completely clears, destroys, and rebuilds the simulation-specific directory structure constructed for each simulation [29]. Finally, each Investigation receives its own date/time-stamped results directory, ensuring that investigations are isolated from one another and internally well-coupled.

\section{MODIFYING STANDARD DESCRIPTIONS OF DOMAIN-SPECIFIC MODELS FOR PROVENANCE AND REUSE}

Ideally, the researcher can use current standardized models of specific entities (such as biophysical models, equations, constants) while not being bound by them. Any modifications to those models should be recorded in the same standardized way for clear provenance and potential reuse. We demonstrated the potential for integration of NeuroManager with ongoing efforts in standardization of computational neuroscience entities [34]. As an example, we flexed

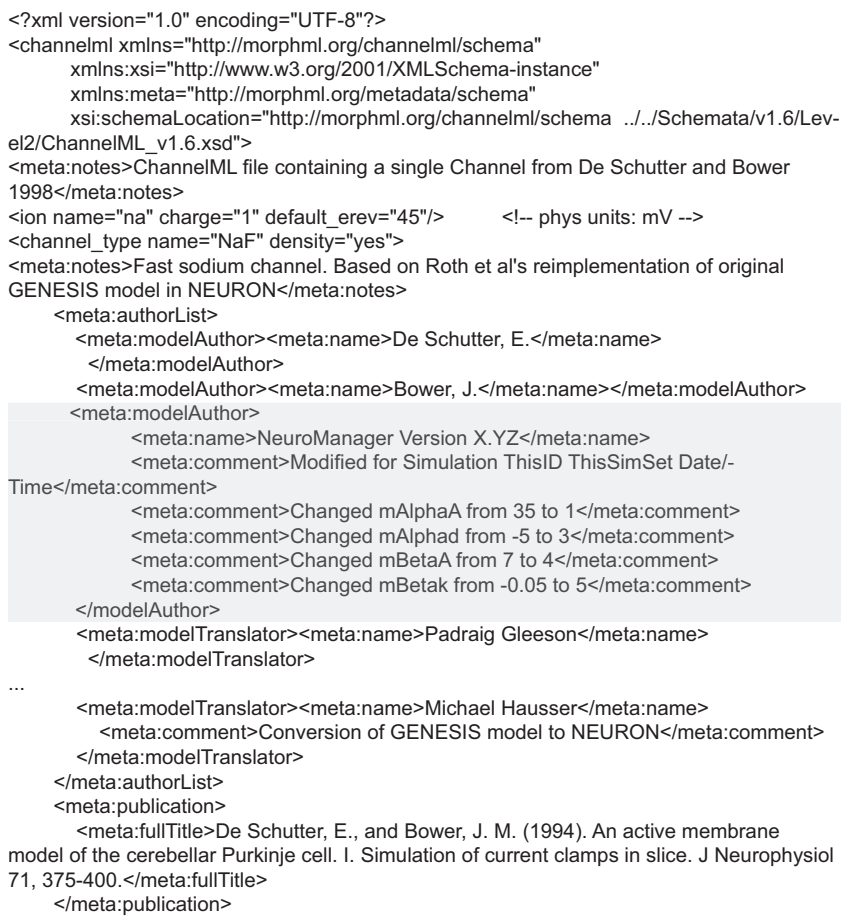

Figure 3: Standardization in NeuroManager. The flexible workflow in NeuroManager allows for integration of XML standardization. Here, NeuroManager adds itself as a model author in the NeuroML description of an ion channel (shaded region). Model source: Gleeson [13]; although this specific model is unavailable, there are many others - see http://www.opensourcebrain.org/search?neuroml_ $\mathrm{DB}=1 \& \mathrm{q}=$ na + naf + sodium .

our fixed workflow to use the NeuroML [14] definition of a biological mechanism called an ion channel. ${ }^{12}$ By utilizing the simulator object hierarchy, NeuroManager was able to access the ion channel properties from the NeuroML file, modify them according to each simulation's needs, and edit the NeuroML file with those modifications as a model translator or author (Figure 3). This ensured that the provenance associated with each simulation, stored in the results directories for that specific simulation, included the XML description of the modified definition, which was then available for purposes of reproducibility. This also allows the researcher to stay standardization-friendly without being handcuffed by current values.

We were also able to describe an entire NeuroManager investigation with an XML file using a custom schema we called "NMSessionML" [34]. This stereotyped the main script that invoked NeuroManager, and allowed the user to merely define the main parameters of the session through the input XML file. Although a little unwieldy in practice, it suggests some of the possibility for reuse and reproducibility that this approach could provide.

\footnotetext{
${ }^{12}$ See https://neuroml-db.org/search_model?q=naf .
} 


\section{ADDING DIMENSIONALITY TO MODEL VERSIONING FOR BETTER MODEL TRAJECTORY, PRESERVATION, AND REPRODUCIBILITY}

Maintaining the trajectory of model development and evolution is an important part of research which is lost when one relies on hacking code and data to make changes. McDougal et al. [22] called out model management as an essential aspect of reproducibility. Adding repository use to daily operations as embodied in Sumatra [11] is an improvement, but not sufficient for complex models. NeuroManager uses object inheritance to capture differences in Virtual Simulators. Since the Virtual Simulator includes a biological model, the researcher can use object inheritance to capture variations and progressions in models as well. This approach allows multi-dimensional versioning independent of the progression of non-model aspects of the code, and allows comparison of models without the "reversion" inherent in traditional software repository management. In other words, the user can compare earlier and later types (or subtypes) of a model on an equal basis.

To illustrate the power of this approach, consider two different models (say A and B) for a neural circuit (Figure 4). Each model requires its own development trajectory and involves biological variables of many types. NeuroManager's approach allows the various versions of models A and B to exist, evolve, and be compared on an equal basis without repository reversion and yet preserves the trajectory of each.

Although currently NeuroManager does not make automated use of repositories like Sumatra, combining the two approaches would bring unprecedented flexibility in model trajectory.

\section{BROADENING COMPUTATIONAL STRATEGIES IN COMPUTATIONAL NEUROSCIENCE}

NeuroManager attempts to broaden three mindsets of computational neuroscience: computational resource type, batch vs interactive, and formal vs informal investigation. Limitations of these mindsets hinder the researcher in time, cost, flexibility, accuracy, and reliability.

The current mindset of computational resource type is simulations are done on institutional or lab-specific clusters, possibly from one's laptop, through manual submission of jobs. This mindset is reinforced by portals such as the Neuroscience Gateway [24], which provides Software as a Service for the computational neuroscience community. NeuroManager broadens this strategy by managing all interactions with external resources, and making it easy to leverage multiple heterogeneous resources simultaneously [35], allowing computational infrastructure to adapt to changing conditions and needs.

The current mindset of batch vs interactive in computational neuroscience is that interactive is more intuitive, and therefore better. However, as is typical with many engineering and scientific graphically-based software packages, one must point, click, and drag multiple windows with hidden parameters to achieve graphical feedback, leading to problems in consistency, accuracy, transparency, provenance, and reproducibility. NeuroManager blurs

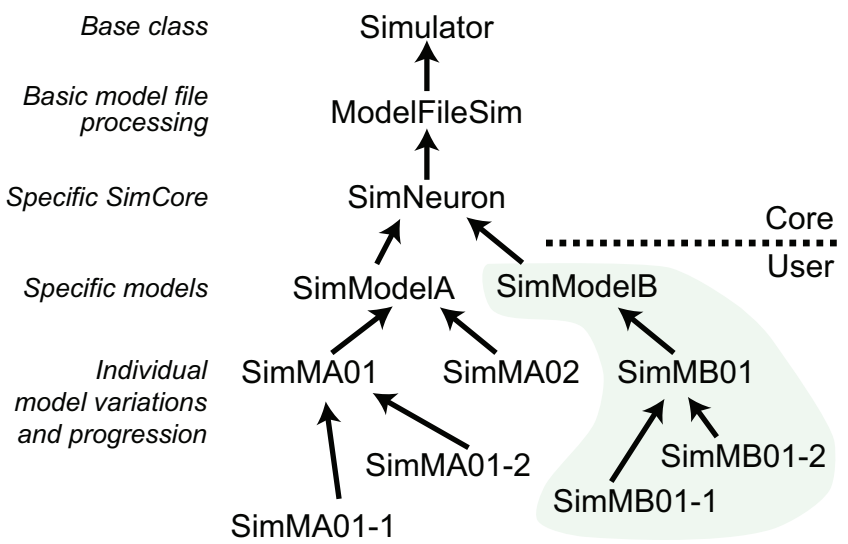

Figure 4: Simulator Inheritance. Left, the general class hierarchy to implement a Simulator. Right, example that implements two independently evolving Simulator types focused on Models A and B. Background shading highlights Model B. Arrows point to the super-class. The implementation of a Simulator can be divided into Core, provided in NeuroManager, and User-defined sections. The core starts with a Simulator base class, then adds a ModelFileSim subclass which allows working with model files; then a specific SimCoreclass adds working properties to process specific models, in this case is SimNeuron that allows running NEURON simulations. Users can then implement specific model subclasses, each of which can have its own variations and trajectory. This approach allows for preservation of various stages of multiple models, all on an equal basis. A variation of this figure is preserved in Stockton and Santamaria [34].

the distinction between interactive and batch approaches by managing experimental database access, simulation job submission, and results data transfer for the user; by bringing hidden parameters out into the open; by making graphical feedback easy; by handling provenance as an automatic process; and even by providing text and email messages with attached figures. In addition, manually difficult simulation-specific changes, such as compiling biomechanisms, become as easy as other parameters, allowing a more comprehensive view of model evolution.

The current mindset of formal vs informal investigation is that provenance, figure production, and recording of activities are best done when one is about to publish, and until then those activities are cumbersome and time-consuming. Often, though, accurate provenance is lost by that time, leading to problems in reproducibility. Ideally accurate provenance is kept continuously, with little user burden. NeuroManager seeks to automate most or all provenance activity and incorporate it into everyday operation as a matter of course. This approach allows informal investigation to be recorded, allows minor changes in code and parameters to become part of a natural process instead of temporary code hacks. Long before publishing, debates about where a particular figure came from are minimized, improving research discussions and decision-making and making research more efficient and reliable. 


\section{CONCLUSIONS}

We incorporated a variety of techniques for provenance and reproducibility into a fixed, yet flexible scientific workflow on multiple computational resource types. We implemented a fixed workflow for transparency while maintaining flexibility, included clouds giving reproducibility of software configuration, sanitized the simulation environment for isolation of simulation-specific parameters and reliability of provenance, demonstrated automatic use of the NeuroML standards for biological models, extended the dimensionality of model trajectory through simulator inheritance, and developed a queryable/publishable investigation database output product. Future efforts should incorporate repository use into the inheritance mechanism, add a Python port for broader scientific and international applicability, and continue integration with other biological standards.

As the need for correct provenance and reproducibility in computational modeling becomes a shared imperative, software support like NeuroManager will become more a part of daily research activities. We hope that the techniques presented here will inform researchers and developers towards that end.

\section{ACKNOWLEDGMENTS}

National Science Foundation (NSFDBI1451032).

\section{REFERENCES}

[1] ABI. 2019. Allen Brain Institute Cell Types Webpage. http:/celltypes.brain-map. org

[2] Amazon. 2019. Amazon Elastic Compute Cloud - API Reference - API Version 2019 https://docs.aws.amazon.com/AWSEC2/latest/APIReference/Welcome.html

[3] Amazon. 2019. Amazon Web Services. https://aws.amazon.com/ Accessed 4 April 2019.

[4] Monya Baker. 2016. 1,500 scientists lift the lid on reproducibility. Nature 533 (2016), 452-454. http://www.nature.com/news/ 1-500-scientists-lift-the-lid-on-reproducibility-1.19970

[5] Cornelia Bargmann, William Newsome, A Anderson, E Brown, K Deisseroth, J Donoghue, P MacLeish, E Marder, R Normann, J Sanes, et al. 2014. BRAIN 2025: a scientific vision. Brain Research through Advancing Innovative Neurotechnologies (BRAIN) Working Group Report to the Advisory Committee to the Director, NIH (2014). https://www.braininitiative.nih.gov/2025/

[6] James M Bower and David Beeman. 2012. The book of GENESIS: exploring realistic neural models with the GEneral NEural SImulation System. Springer Science \& Business Media.

[7] Lucian Carata, Sherif Akoush, Nikilesh Balakrishnan, Thomas Bytheway, Ripduman Sohan, Margo Seltzer, and Andy Hopper. 2014. A Primer on Provenance. Commun. ACM 57, 5 (May 2014), 52-60. https://doi.org/10.1145/2596628

[8] ChameleonCloud. 2019. Chameleon Cloud: A configurable experimental environment for large-scale cloud research. https://www.chameleoncloud.org/

[9] Colin D. Chapman, Christian Benedict, and Helgi B. Schiöth. 2018. Experimenter gender and replicability in science. Science Advances 4, 1 (jan 2018), e1701427. https://doi.org/10.1126/sciadv.1701427

[10] Robert L Clay. 2016. Engineering sciences workflow in the Sandia Analysis Workbench [PowerPoint]. Technical Report. Sandia National Lab.(SNL-CA), Livermore, CA (United States).

[11] Andrew Davison. 2012. Automated capture of experiment context for easier reproducibility in computational research. Computing in Science \& Engineering 14, 4 (2012), 48-56.

[12] Juliana Freire, Norbert Fuhr, and Andreas Rauber. 2016. Reproducibility of dataoriented experiments in e-science (dagstuhl seminar 16041). In Dagstuhl Reports, Vol. 6. Schloss Dagstuhl-Leibniz-Zentrum fuer Informatik.

[13] Padraig Gleeson. 2015. NaF Channel XML Description Website. https://neuroml. org/NeuroMLValidator/NeuroMLFiles/Examples/ChannelML/NaF_Chan.xml

[14] Padraig Gleeson, Sharon Crook, Robert C. Cannon, Michael L. Hines, Guy O. Billings, Matteo Farinella, Thomas M. Morse, Andrew P. Davison, Subhasis Ray, Upinder S. Bhalla, Simon R. Barnes, Yoana D. Dimitrova, and R Angus Silver 2010. NeuroML: A language for describing data driven models of neurons and networks with a high degree of biological detail. PLoS Computational Biology 6, 6 (Jun 2010), e1000815. https://doi.org/10.1371/journal.pcbi.1000815
[15] Dan FM Goodman and Romain Brette. 2009. The Brian simulator. Frontiers in neuroscience 3 (2009), 26.

[16] Nikolay Grozev and Rajkumar Buyya. 2014. Inter-Cloud architectures and application brokering: taxonomy and survey. Software: Practice and Experience 44, 3 (2014), 369-390.

[17] Cengiz Günay, Jeremy R. Edgerton, Su Li, Thomas Sangrey, Astrid A. Prinz, and Dieter Jaeger. 2009. Database analysis of simulated and recorded electrophysiological datasets with PANDORA's toolbox. Neuroinformatics 7, 2 (Jun 2009), 93-111. https://doi.org/10.1007/s12021-009-9048-z

[18] Michael. L. Hines and Nicholas. T. Carnevale. 2001. NEURON: A Tool for Neuroscientists. Neuroscientist 7, 2 (Apr 2001), 123-135.

[19] David N. Kennedy, Sanu A. Abraham, Julianna F. Bates, Albert Crowley, Satrajit Ghosh, Tom Gillespie, Mathias Goncalves, Jeffrey S. Grethe, Yaroslav O. Halchenko, Michael Hanke, Christian Haselgrove, Steven M. Hodge, Dorota Jarecka, Jakub Kaczmarzyk, David B. Keator, Kyle Meyer, Maryann E. Martone, Smruti Padhy, Jean-Baptiste Poline, Nina Preuss, Troy Sincomb, and Matt Travers. 2019. Everything Matters: The ReproNim Perspective on Reproducible Neuroimaging. Frontiers in Neuroinformatics 13 (feb 2019). https: //doi.org/10.3389/fninf.2019.00001

[20] Johan N. Lundström and Mats J. Olsson. 2005. Subthreshold amounts of social odorant affect mood, but not behavior, in heterosexual women when tested by a male, but not a female, experimenter. Biological Psychology 70, 3 (dec 2005), 197-204. https://doi.org/10.1016/j.biopsycho.2005.01.008

[21] Mathworks. 2015. MATLAB Runtime. http://www.mathworks.com/products/ compiler/mcr/

[22] R. McDougal, A. Bulanova, and W. Lytton. 2016. Reproducibility in Computational Neuroscience Models and Simulations. IEEE Transactions on Biomedical Engineering PP, 99 (2016), 1-1. https://doi.org/10.1109/TBME.2016.2539602

[23] MySQL. 2017. MySQL Website. https://www.mysql.com/

[24] NSG. 2019. Neuroscience Gateway Website. https://www.nsgportal.org/

[25] Rackspace. 2019. Rackspace Website. https://www.rackspace.com/en-us

[26] Michael Smit, Przemyslaw Pawluk, Bradley Simmons, and Marin Litoiu. 2012. A web service for cloud metadata. In Services (SERVICES), 2012 IEEE Eighth World Congress on. IEEE, 361-368.

[27] Daniel Stenberg. 2017. Everything cURL. https://curl.haxx.se/

[28] Joel R Stiles and Thomas M Bartol. 2001. Monte Carlo Methods for Simulating Realistic Synaptic Microphysiology Using MCell. In Computational Neuroscience: Realistic Modeling for Experimentalists, Erik De Schutter (Ed.). CRC Press, Boca Raton, FL, Chapter 4, 87-127.

[29] David B. Stockton. 2015. NeuroManager 0.961 User and Programmer's Guide. https://github.com/SantamariaLab/NeuroManager/blob/master/ UserGuide/UserGuideRev6PostReview.pdf

[30] David B. Stockton. 2015. NeuroManager: A workflow analysis based... - Supplemental Material. (2015). http://journal.frontiersin.org/file/downloadfile/156632 supplementary-materials_presentations_1_pdf/octet-stream/Presentation\%201. PDF/1/156632

[31] David B. Stockton. 2016. NeuroManager User and Programmer's Guide - 0.980 Addendum. https://github.com/SantamariaLab/NeuroManager/blob/master/ UserGuide/NMUGAddendum.pdf

[32] David B. Stockton. 2016. NeuroManager User and Programmer's Guide - 0.985 Addendum ("One Compile" Upgrade). https://github.com/SantamariaLab/ NeuroManager/blob/master/UserGuide/UGOneCompileAddendum.pdf

[33] David B. Stockton. 2017. NeuroManager User and Programmer's Guide - 0.990 Addendum ("Investigation Database" Upgrade). (2017). https://github.com/ SantamariaLab/NeuroManager/blob/master/UserGuide/NMUGAddendum.pdf

[34] David Bruce Stockton and Fidel Santamaria. 2015. NeuroManager: A workflow analysis based simulation management engine for computational neuroscience. Frontiers in Neuroinformatics 9, 24 (2015). https://doi.org/10.3389/fninf.2015. 00024

[35] David B. Stockton and Fidel Santamaria. 2016. Automating NEURON Simulation Deployment in Cloud Resources. Neuroinformatics (Sep 2016). https://doi.org/ 10.1007/s12021-016-9315-8

[36] David B. Stockton and Fidel Santamaria. 2017. Integrating the Allen Brain Institute Cell Types Database into Automated Neuroscience Workflow. Neuroinformatics (02 Aug 2017). https://doi.org/10.1007/s12021-017-9337-x

[37] Univa. 2019. Univa Grid Engine. http://www.univa.com/resources/files/ gridengine.pdf

[38] Nicole A. Vasilevsky, Matthew H. Brush, Holly Paddock, Laura Ponting, Shreejoy J. Tripathy, Gregory M. Larocca, and Melissa A. Haendel. 2013. On the reproducibility of science: unique identification of research resources in the biomedical literature. Peerf 1 (2013), e148. https://doi.org/10.7717/peerj.148

[39] Andy B Yoo, Morris A Jette, and Mark Grondona. 2003. SLURM: Simple Linux Utility for Resource Management. In fob Scheduling Strategies for Parallel Processing. Springer, 44-60. 